\title{
Motivación en el trabajo: viejas teorías, nuevos horizontes
}

\author{
Carlos Arrieta-Salas \\ Escuela de Psicología, Universidad de Costa Rica \\ Dirección Postal : 11501-2060 \\ Ce: carlos.arrieta@ucr.ac.cr \\ José Navarro-Cid \\ Departamento de Psicología Social, Universidad de Barcelona. \\ Dirección postal: Paseo Valle de Hebrón, 171 \\ 08035 Barcelona, España \\ Ce: j.navarro@ub.edu
}

\begin{abstract}
Resumen. A pesar de tratarse de uno de los constructos centrales en psicología de las organizaciones y del trabajo, la investigación y el desarrollo de nuevas teorías en motivación laboral han disminuido considerablemente en los últimos años. Varios autores atribuyen esta situación al dominio ejercido por las teorías clásicas y plantean la necesidad de desarrollar nuevos enfoques teóricos y nuevas estrategias metodológicas con el fin de revitalizar el campo adecuándolo a las condiciones vigentes en la actualidad. En este trabajo se analizan algunas de las críticas más importantes dirigidas a las teorías de la motivación -principalmente a las teorías de procesos-, prestando especial atención a aquélla que indica que estas teorías han fallado sistemáticamente al analizar los efectos potenciales del tiempo sobre los componentes más importantes de la teoría. Sobre la base de esta crítica la tesis principal que se sostiene y defiende en este artículo es que la motivación laboral apenas ha sido estudiada como proceso. Por otra parte, se presentan enfoques alternativos para el estudio de la motivación centrados principalmente en el estudio del comportamiento dinámico y complejo del fenómeno.
\end{abstract}

Palabras clave: motivación en el trabajo, tiempo, complejidad.

Abstract. Despite of being one of the central concepts in organizational psychology, research and development of new theories in work motivation have decreased considerably in recent years. Several authors suggest that this situation is the result of the dominating role that traditional theories of work motivation have exerted, and they indicate the necessity to develop new theoretical approaches and methodologies to revitalize the field. This article analyzes one of the most important criticisms directed toward the theories of processes and emphasizes on those criticisms that underline that work motivation theories generally failed to incorporate time as an important variable affecting the most relevant components of the theory and the people's motivation. Our main conclusion is that work motivation has barely been studied as a process. Therefore, we present alternative approaches for the study of work motivation as a dynamic and complex phenomenon.

Key Words: Work motivation, time, complexity. 


\section{Introducción}

Tradicionalmente la motivación en el trabajo ha constituido un proceso de medular importancia tanto desde la perspectiva de la gestión organizacional como desde la perspectiva de la investigación (c.f. Peiró, 1990; Steer, Mowday \& Saphiro, 2004; Thierry, 1998). En concordancia con lo anterior, resulta significativo el número de teorías que se han desarrollado al respecto y la gran cantidad de investigaciones que se han llevado a cabo hasta el momento. El interés principal de estos esfuerzos se ha orientado hacia cuatro fines, a saber, identificar los componentes centrales de la motivación en el trabajo, elaborar modelos adecuados para investigarlo, contrastar empíricamente los modelos y teorías propuestos y derivar a nivel pragmático resultados de interés para la gestión organizacional.

Ahora bien, a pesar de la importancia que se le reconoce a este constructo, algunos autores cuestionan si en la actualidad continúa manteniendo la misma relevancia en virtud principalmente de tres cuestiones: primera, del estancamiento que perciben en términos de la generación de nuevas teorías de la motivación a la luz de las condiciones vigentes en la actualidad (Steers, Mowday \& Shapiro, 2004); segunda, de un descenso significativo de la investigación llevada a cabo en relación con este tema y del "olvido" del constructo a la hora de realizar investigaciones sobre este fenómeno debido al énfasis puesto en el estudio de relaciones muy específicas entre variables (Ambrose \& Kulik, 1999); y, tercera, de las transformaciones que están experimentando las organizaciones $-\mathrm{y}$ los procesos que en ellas tienen lugar- como resultado de los cambios que se están dando a nivel societal (globalización) y como resultado del impacto de los avances en materia del desarrollo de nuevas tecnologías de la producción y de la comunicación (Cf. Andriessen, 1994, 2002; Morgan, 1999; Prieto, Zornoza \& Peiró, 1996; Prieto, Zornoza, Orengo \& Peiró, 1996; Zornoza, Orengo \& Peiró, 2000).

En relación con este último aspecto, autores como Rousseau (1997) y Wilpert (1995), son del criterio de que la psicología de las organizaciones y del trabajo está experimentando una suerte de renovación paradigmática debida a una serie de confrontaciones teórico-epistemológicas que se están suscitando en la actualidad en este campo y que se expresa, entre otras cosas, en dos indicadores que consideramos significativos, a saber: 1) Los cambios que se pueden observar fácilmente en las políticas editoriales de las revistas científicas. Estas tradicionalmente han establecido las líneas de investigación "duras" en términos de lo que consideran investigación provechosa para el desarrollo del conocimiento científico. Más bien se nota en este momento cierta "flexibilización" y "apertura" hacia el desarrollo de nuevas perspectivas y enfoques teórico metodológicos y un interés por dirigir la atención hacia 
otras perspectivas ya existentes, las cuales hasta hace un tiempo no gozaban de un nivel de legalidad significativo en ese contexto, por ejemplo, aproximaciones construccionistas, aproximaciones de teoría de la complejidad. 2) En el cuestionamiento de la tradicional forma de investigar y que se observa en una cantidad significativa de artículos publicados en los que, por un lado, se revisa críticamente el monismo metodológico que ha imperado por mucho tiempo sobre todo en términos de la búsqueda de causalidad desde una perspectiva lineal y, por otro lado, en la toma de conciencia con respecto al "descuido" de la dimensión temporal, tan importante si se quiere conocer realmente el dinamismo de los fenómenos que se estudian (c.f. Fried \& Slowik, 2004; George \& Jones, 2000; McGrath \& Tschan, 2004; Mitchell \& James, 2001; Morgan, 1999; Munné, 1995; Roe, 2005a , 2005b).

En el año 2004 la Academy Management Review publicó un número especial dedicado exclusivamente a analizar el futuro de la investigación en motivación laboral. Este forum fue convocado con la consigna de que sirviera como un espacio de reflexión en torno a una serie de interrogantes que Steers, Mowday y Shapiro (2004) resumen de la siguiente manera: ¿cuál es el futuro de las teorías de motivación laboral?, ¿cuáles son las cuestiones críticas que deben ser planteadas si se quiere que el campo progrese?, ¿cuál es la agenda de investigación futura?, ¿cómo pueden ser ampliados o modificados los actuales modelos de motivación laboral de modo que sigan siendo relevantes en el futuro?, ¿dónde están los modelos enteramente nuevos de motivación necesitados para comprender la conducta del empleado y el desempeño laboral en las organizaciones contemporáneas?, etc.

En conjunto, los artículos publicados en este forum, coinciden en que es necesario trazar nuevas líneas de desarrollo a nivel teórico y metodológico, una vez puestas al descubierto las limitaciones más importantes de la investigación en motivación laboral. Estas limitaciones giran a nuestro entender en torno de una crítica medular, formulada por Fried y Slowik (2004), a saber, que las teorías de la motivación han fallado sistemáticamente al dirigir los efectos potenciales del tiempo sobre los componentes más importantes de la teoría, provocando con ello un empobrecimiento de la teoría misma. La formulación de estos autores coincide con las apreciaciones de otros autores que anteriormente se habían referido a este mismo tema (c.f. George \& Jones, 2000; Kanfer, 1990; Mitchell \& James, 2001; Navarro, 2001). La idea central aquí es que difícilmente se puede llegar a conocer el dinamismo de un fenómeno, si no se tiene en cuenta de manera explícita cómo es su comportamiento a lo largo del tiempo desde una perspectiva de proceso. Tal y como lo plantean George y Jones (2000) dado que la motivación es un fenómeno creado subjetivamente, los constructos existentes quedan en deuda en cuanto a su capacidad para dar cuenta de la 
naturaleza cambiante y subjetiva de este fenómeno, al estar limitados a una perspectiva del tiempo estándar o tiempo reloj. Lo que es necesario, dicen George y Jones, es una concepción teórica integral que tome en cuenta cómo el pasado multifacético y la variedad de formas en las cuales la gente anticipa el futuro influencian las fuerzas psicológicas en el presente que subyacen a la motivación.

En el caso de la motivación en el trabajo, la definición más aceptada es aquella que la conceptualiza como fuerzas psicológicas que operan desde dentro de las personas y que determinan la dirección de las conductas emitidas, de los niveles de esfuerzo y de persistencia cuando la conducta no está sujeta a fuertes presiones y limitaciones situacionales (Kanfer, 1990; Campbell \& Pritchard, 1976; Vroom, 1964). Se trata, tal y como lo señalan Latham y Pinder (2005) y Latham y Ernst (2006), de un proceso psicológico resultado de la interacción entre el individuo (necesidades, valores, cogniciones, intereses, metas, etc.) y el ambiente (condiciones de trabajo, tipo de tarea, retroalimentación, presión temporal, etc.). De lo anterior, resulta evidente que la relación dinámica de los procesos implicados, su carácter subjetivo y la importancia de los factores situacionales juegan un papel central a la hora de investigar este fenómeno y como condición para lograrlo los diseños de investigación y las metodologías empleadas deben ser sensibles a las vicisitudes del fenómeno a lo largo del tiempo. Por ejemplo, si sabemos que la motivación en el trabajo muestra variaciones importantes a lo largo de la vida de una persona, incluso variaciones al cabo de una semana de trabajo o de un día, se necesitaría de diseños de investigación que fueran capaces de captar estos cambios y no presupusieran, de entrada, que la motivación se mantiene estable a lo largo del tiempo.

Dada la envergadura de las críticas dirigidas a la investigación llevada a cabo en torno a la motivación en el trabajo y a las teorías desarrolladas hasta el momento, en este trabajo presentamos una serie de reflexiones sustentadas a partir de una revisión sistemática de los artículos más relevantes publicados en los últimos quince años por autores de reconocida trayectoria en el campo y que aparecen consignados en la base de datos PsycINFO de la American Psychological Association (artículos de investigación, artículos de revisión, monográficos, foros, tesis doctorales, etc.). Los términos empleados para la búsqueda incluyeron palabras clave como motivación, motivación en el trabajo, dinámica, longitudinal, tiempo, temporalidad, cambio, complejidad, entre otras.

Las reflexiones derivadas de esta revisión las articulamos en torno a una tesis central: hasta ahora la motivación laboral apenas ha sido estudiada como proceso. Un enunciado de esta naturaleza puede resultar sorprendente en especial si se tiene en cuenta que las teorías de la motivación más potentes desarrolladas hasta el momento (teorías de las expectativas o VIE, teoría del establecimiento de metas, teorías de la justicia organizativa y teoría de la 
autoeficacia) han sido clasificadas precisamente bajo la etiqueta "teorías de proceso" (Munduate, 1994; Navarro, 2001; Salanova, Hontangas \& Peiró, 1996; Thierry, 1998). Sin embargo, a nuestro juicio, la noción de proceso sobre la que se asientan gran parte de estas teorías resulta bastante limitada, lo que a su vez reduce considerablemente su capacidad para estudiar el comportamiento dinámico de la motivación en el trabajo tal y como es experimentada por los trabajadores.

\section{Estado actual del campo de la motivación laboral}

Las teorías de la motivación han sido tradicionalmente clasificadas en dos grupos, a saber, las teorías centradas en los contenidos de la motivación y las centradas en los procesos (c.f. Campbell, Dunnette, Lawler \& Weick, 1970; Kanfer, 1990; Navarro, 2001; Salanova, Hontangas \& Peiró, 1996). Las teorías de contenido se caracterizan por conceptualizar e investigar la motivación como un fenómeno relativamente estático. Se interesan por determinar el por qué de la conducta motivada y basan sus planteamientos en una serie de necesidades cuya anticipación de satisfacción determinarán el nivel de motivación que experimenta la persona. Las teorías de procesos, por su parte, ponen su énfasis en el proceso mediante el cual una conducta se convierte en conducta motivada. En este sentido se interesan por analizar los procesos, en su mayor parte de tipo cognitivo, involucrados en la dirección, intensidad y persistencia de la conducta motivada y destacan la necesidad de establecer relaciones causales a través del tiempo y de las situaciones que acontecen en el contexto laboral.

Dado que son las teorías de proceso las que en la actualidad gozan de un mayor reconocimiento en virtud del poder explicativo que han mostrado a lo largo de una gran cantidad de pruebas y contrastaciones empíricas y debido a la menor investigación llevada a cabo desde las teorías de contenido, la valoración que haremos del campo en este apartado y a lo largo de este artículo versará principalmente en torno a éstas. Dicho lo anterior, según Steer, Mowday y Shapiro (2004) a partir de la década de los noventa se nota un sensible declive en la investigación llevada a cabo sobre motivación laboral. A juicio de estos autores basta hacer revista de los artículos aparecidos en las principales publicaciones científicas relativas a temas del comportamiento organizacional para percatarse de la disminución de pruebas empíricas o de aplicaciones de las teorías existentes. Asimismo, una revisión de los más recientes libros de texto sobre este tema revela que la mayoría de las teorías discutidas datan de los años sesenta y setenta con fugaces intentos por desarrollar nuevos aportes (véase, por ejemplo, un manual clásico como el de Robbins, 2003).

En 1999 Ambrose y Kulik llevaron a cabo una revisión del comportamiento de la investigación en motivación laboral en el período 
comprendido entre 1990 y 1997 a partir del análisis de más de 200 estudios aparecidos en diversas revistas especializadas. En términos generales, los autores concluyen que durante este período se realizaron muy pocos aportes teóricos sustanciales debido en buena parte a que la mayor parte de la investigación estuvo supeditada al dominio de lo que ellos denominan las "viejas" teorías de la motivación. A juicio de los autores, esta situación provocó que la mayor parte de la investigación realizada se hiciera con el fin de someter a contrastación los modelos teóricos existentes o, mejor dicho, de investigar fragmentadamente componentes muy específicos de dichos modelos: "Organizational behavior research has largely abandoned the concept of "motivation" and has replaced this broad concept with more specific measures of employee behavior (e.g., task performance, organizational citizenship behaviors)... That is, researchers did not intend to study 'motivation" (Ambrose \& Kulik, 1999, p.278).

Frrente a este estado de cosas los autores se preguntan, no sin asombro, cómo es posible que los investigadores hayan escrito artículos empíricos usando las teorías de la motivación sin usar el constructo central de motivación y optando por estudiar aspectos muy específicos relacionados con este constructo.

Latham y Pinder (2005) publicaron un artículo de revisión en el que analizaron la producción comprendida entre los años 1995 y 2003. Estos autores no refieren explícitamente a que durante dicho período la investigación realizada haya orbitado hegemónicamente en torno de las teorías clásicas, pero tampoco en sus conclusiones mencionan la aparición de aportes sustancialmente novedosos. A lo largo del artículo se dan a la tarea de enumerar lo que consideran los cambios más importantes, los que en esencia están referidos a la apertura de nuevas temáticas de investigación: a nivel del sujeto (necesidades, valores, cognición -metas-, afecto -emocionesy conducta) y a nivel organizacional y contextual (características del trabajo, ajuste persona-organización, cultura nacional). Sin embargo, pocos o ningún comentario se encuentra en relación con asuntos relativos a cuestiones epistemológicas, a la elaboración de nuevos modelos o diseños de investigación o la propuesta de nuevas teorías. Con respecto a este último aspecto, los autores señalan que las teorías dominantes en la última década han sido la teoría del establecimiento de metas, la teoría social cognitiva y la teoría de justicia organizacional, lo que pone en evidencia precisamente el anclaje en propuestas teóricas desarrolladas hace más de una década.

De los antecedentes apuntados hasta aquí se puede colegir que en el campo de la investigación en motivación en el trabajo se percibe una suerte de estancamiento en la producción de nuevos aportes sustanciales, lo que ha provocado que a nivel de la formulación de nuevos enfoques el conocimiento acumulado hasta el momento se haya mantenido más o menos en las mismas condiciones desde la década de los noventa. 
En relación con este estado de cosas, Steers, Mowday y Shapiro (2004) se cuestionan acerca de si esto se debe a que se ha logrado crear un corpus de conocimiento completo y acabado sobre el tema como resultado de las múltiples contrastaciones empíricas a las que se han sometido las teorías existentes, o de si más bien el tópico ha perdido interés como tema de investigación. Para dichos autores ninguna de las dos alternativas resulta atinada por cuanto consideran que un tópico como éste continúa siendo de vital importancia a nivel teórico y aplicado; más bien creen que la supeditación de la investigación al dominio de las mismas perspectivas ha provocado, entre otras cosas, reiteraciones y recurrencias alrededor de las mismas ideas y enfoques limitando con ello su desarrollo. Y en virtud de ello abogan por una revitalización del campo especialmente en un momento en que las organizaciones y el mundo del trabajo cambian dramáticamente interpelando con ello a los investigadores, académicos y gestores "...to develop the breakthrough ideas that can push us to the next level of understanding"" (Steers, Mowday \& Shapiro, 2004, p. 383)

\section{Acerca de las críticas más significativas dirigidas a las teorías de procesos en motivación laboral}

Una serie de condiciones interpelan en la actualidad el campo de la investigación en comportamiento organizacional propiciando la emergencia de confrontaciones paradigmáticas allí donde las cosas se habían mantenido más o menos estables por mucho tiempo. La investigación en motivación laboral obviamente no escapa a los efectos de dichas tensiones.

Una lectura crítica ha llevado a varios autores (c.f. Mowday \& Sutton, 1993; Rousseau, 1997; Wilpert, 1995) a tomar consciencia de que estas confrontaciones paradigmáticas conducen al cuestionamiento, en mayor o menor grado, de las bases mismas del quehacer investigativo en lo concerniente tanto al modelo de investigación imperante como a sus estrategias metodológicas. Asimismo, advierten que las condiciones históricas prevalecientes en los últimos quince años comportan características muy diferentes a las de épocas anteriores y de que esto debe ser necesariamente tomado en cuenta a la hora de definir cualquier agenda de investigación a futuro. Por ejemplo, señalan que los cambios que se están dando en cuanto a la forma de concebir las organizaciones y el mundo del trabajo (las compañías se hacen más pequeñas y se expanden a la vez, la fuerza de trabajo es más diversa y con demandas y necesidades divergentes, las tecnologías de la información y de la producción han cambiado la forma

1 “...desarrollar ideas avanzadas que empujen hacia un nuevo nivel de entendimiento" 
de trabajar, etc.) afectan en forma directa al objeto de estudio y ello a su vez a la investigación y a la producción de teoría. A su entender, el tradicional modelo de organización mecanicista (rígido y estructurado en función de las premisas del control y la predicción) ha ido debilitándose en la misma medida en que han empezado a tomar fuerza formas alternativas basadas en perspectivas dinámicas que ven a las organizaciones -y los procesos que en ellas acontecen- como entidades en proceso organizadas de manera flexible. Nociones como organizing (Weick, 2001) y autoorganización (Morgan, 1999) empiezan a aflorar como concepciones significativas en un nuevo orden discursivo, asimismo se empiezan a desarrollar nuevas formulaciones teóricas y a prestársele atención a otras que ya existían pero que no eran tomadas en cuenta en vista de que no gozaban de un orden de legalidad reconocido. En palabras de Rousseau (1997): "we might need to acknowledge that changes in firms are profound enough to alter further basic assumptions on which the field is based. In any case, a new era in organizational behavior appears to be in the making"2 (p. 537).. En relación con lo anterior y en el caso de la motivación en el trabajo, Steers, Mowday y Shapiro (2004) son del criterio que "...the time has come to redirect our intellectual energies into discovering new models -and research toward new models- of work motivation and job performance commensurate with this new era"3 (p. 384).

En concordancia con las recomendaciones, cierto consenso parece ir gestándose con respecto a que es necesario dotar a la teoría de mayor diversidad, dinamismo y profundidad prestándole especial importancia a la dimensión temporal. En los últimos tiempos han surgido una sucesión de críticas en las que se señala que la investigación realizada ha descuidado o tratado con ligereza un asunto de vital importancia para la comprensión adecuada del fenómeno investigado como es el tema del tiempo (c.f. Ancona, Goodman \& Tushman, 2001; Ancona, Okhuysen \& Perlow, 2001; Bluedorn \& Denhardt, 1988; Fried \& Slowik, 2004; George \& Jones 2000; McGrath \& Kelly, 1986; McGrath \& Tshan, 2004; Mitchell \& James, 2001; Mosakowski \& Earley, 2000; Zaheer, Albert \& Zaheer, 1999). Los autores coinciden en señalar que la incorporación explícita de la dimensión temporal a nivel teórico y metodológico -mal tratada en buena parte de la investigación incluida la realizada en el campo de la motivación- contribuiría significativamente a enriquecer la investigación, fortaleciéndola frente a las críticas que cuestionan su validez debido a las debilidades que quedan a

\footnotetext{
2 "necesitaríamos reconocer que los cambios en las empresas son lo suficientemente profundos como para alterar las asunciones básicas sobre las que se apuntala el campo. En cualquier caso, parece que se está gestando una nueva era en el comportamiento organizacional”

3 “...ha llegado el momento de redirigir nuestras energías intelectuales hacia el descubrimiento de nuevos modelos $-\mathrm{y}$ a la investigación de nuevos modelos- de motivación laboral y de desempeño acordes con esta nueva era"
} 
descubierto cuando las teorías construidas se someten a contrastación o falsación desde una perspectiva temporal y teniendo como eje articulador las características fenoménicas del objeto de estudio.

Sobre la premisa del descuido de la dimensión temporal una revisión de la literatura permite observar las debilidades de las teorías de la motivación formuladas hasta el momento, especialmente las relativas al estudio dinámico del fenómeno. Kanfer (1990) y Navarro (2001) han hecho a nivel metodológico importantes críticas a estas teorías por considerar que en general limitan la noción de proceso al establecimiento de relaciones episódicas entre variables ya que las mediciones que proponen con el fin de capturar la dinámica del fenómeno, resultan más bien medidas sincrónicas fundamentadas en la creencia de que fenómenos como el de marras se caracterizan esencialmente por ser estables y equilibrados. A nivel teórico, Navarro (2001) ha señalado que la limitación más importante de estas teorías estriba en que conceptualizan la motivación como un fenómeno lineal cuando en realidad se trata de un fenómeno cuyo comportamiento dista de ser estable y consistente a lo largo del tiempo. Así, en un trabajo posterior, Navarro, Arrieta y Ballen (2007) señalan que la mayor parte de las teorías elaboradas hasta ahora apenas han abordado la motivación en su aspecto dinámico, es decir, como proceso que varía a lo largo del tiempo, y en su lugar se han interesado por los aspectos más inmediatos que afectan a la elección, intensidad y persistencia del comportamiento que calificamos de motivado.

En concordancia con lo anterior, en el año 2001 la Academy of Management Review convocó un forum dedicado al tema del tiempo en la investigación con el objetivo explícito de explorar el significado del tiempo y de los marcos conceptuales abocados al tema de la temporalidad en las organizaciones. Haciendo eco de las críticas formuladas por Mitchell y James (2001), los diferentes artículos incluidos en este forum plantean que el tratamiento inadecuado de la dimensión temporal en la investigación se debe, entre otras cosas, a un asunto de comodidad ya que resulta más sencillo investigar la relación entre variables si se considera que entre ellas existe una relación exclusiva, estable y lineal. Y ello en buena parte se debe también a que cuando se hace referencia a los procesos y dinámicas de las organizaciones se ha partido tácitamente del supuesto de que se trata de estados que cambian poco y que en general tienden a buscar el equilibrio y la estabilidad. De ahí que las investigaciones busquen establecer relaciones de causalidad entre variables medidas de manera sincrónica (diseños transversales) y cuando se utilizan diseños longitudinales se consideran no más allá de tres o cuatro registros a lo largo del tiempo. De acuerdo a una estimación hecha por Wright (2002) más de un 90\% de los artículos de investigación publicados en la revista Organizational Behavior son transversales y de naturaleza no basada en el tiempo. 
Por ejemplo Roe (2005a) señala que en los resultados de un análisis de contenido hecho a partir de los títulos y de los abstracts de los artículos publicados en las revistas Journal of Applied Psychology, European Journal of Work y Organizational Psychology, durante los años 2003-2004 aparecen muy escasas referencias a términos como "tiempo", "temporalidad", "desarrollo" y "cambio". Tampoco aparecen referencias a momentos de medida y duración (años, meses, etc.). Asimismo, cuando el autor revisó más detenidamente aquellos artículos en los que aparecieron términos como desarrollo, crecimiento y dinámicas descubrió que en ellos prevalecía, no obstante, una concepción estática. En cuanto a estudios longitudinales llevados a cabo en psicología de las organizaciones y del trabajo, Roe (2005a) señala que una revisión seria pone en evidencia, primero que todo, que los investigadores difícilmente se interesan en el tiempo como tal y los diseños de sus investigaciones son primariamente herramientas para determinar la dirección de la causalidad o secuencialidad de las relaciones entre variables.

A nuestro entender, resulta un contrasentido, teórica y metodológicamente hablando, pretender capturar el dinamismo de un proceso a partir de una concepción estática de los fenómenos y a partir de un repertorio tan limitado de registros y observaciones de su comportamiento. El estudio la motivación como proceso dinámico, supone registrar constantemente su comportamiento a lo largo del tiempo de modo que sea posible visualizar los cambios que va sufriendo en su devenir como resultado de los intercambios inevitables que se dan entre el sujeto y el entorno. La medición episódica del fenómeno, por el contrario, lo vuelve artificial al registrar características que podría efectivamente comportar en un espaciotiempo determinado pero que no necesariamente resultan representativas del comportamiento general del fenómeno desde una perspectiva diacrónica. A este respecto, George y Jones (2000) manifiestan categóricamente que una conceptualización de la motivación debe contener referencias a su naturaleza inherentemente subjetiva y cambiante, por lo que las definiciones no deberían estar sometidas al dominio de la concepción del tiempo estándar.

En virtud de lo anterior, una propiedad fundamental que debiera ser contemplada en los modelos e investigaciones sobre motivación es que se trata de un fenómeno que se realimenta constantemente tanto en función de las fuerzas psicológicas que operan desde dentro del sujeto como de las condiciones que prevalecen en su entorno. Esta propiedad de realimentación lamentablemente es poco tomada en cuenta a la hora de investigar ya que, como se ha expresado a lo largo de este apartado, el factor tiempo, visto desde esta perspectiva, no ha jugado un papel relevante en la explicación del comportamiento y devenir de los fenómenos estudiados. Tal y como lo menciona Navarro (2001), en psicología de las organizaciones y del trabajo se habla de procesos psicológicos como por ejemplo la motivación, el conflicto $\mathrm{y}$ ambigüedad de rol y de procesos psicosociales como por ejemplo el 
liderazgo o conflicto, desde una perspectiva muy estática, es decir, como si fueran fenómenos o procesos regulares en su conformación y en sus expresiones fenoménicas; en el mejor de los casos cuando son estudiados desde una perspectiva menos estática su comportamiento es sometido a un path analysis (análisis de trayectorias) que incluye alguna relación de retroalimentación. Para articular una auténtica visión de proceso los fenómenos estudiados deberían ser considerados como procesos dinámicos que cambian sutil y/o drásticamente a lo largo del tiempo. Además debiera tenerse en cuenta que la intensidad del cambio muchas veces no guarda una relación de proporcionalidad con respecto a las consecuencias que podría tener sobre el objeto.

Desde nuestra perspectiva, aquí radica una de las limitaciones más importantes de la investigación psicosocial en general y de la investigación en motivación laboral en particular y, por tanto, una de las limitaciones más importantes de la investigación llevada a cabo a la luz de las teorías de procesos. Las teorías clasificadas bajo esta denominación cumplen parcialmente su propósito en la medida en que la concepción de temporalidad que les es intrínseca es bastante limitada. Por ejemplo, la teoría de las expectativas o VIE (c.f. George \& Jones, 2000; Vroom, 1964) reconoce explícitamente que su énfasis está en el aquí y el ahora con lo cual se define a sí misma como una teoría esencialmente ahistórica en tanto los antecedentes y los consecuentes del comportamiento no juegan realmente un papel significativo. Al considerar el comportamiento de sus componentes (valencia, expectativa e instrumentalidad) no toma en cuenta el pasado (v.gr. aprendizajes previos) ni el futuro (v.gr. cambios venideros en las condiciones organizativas) ni cómo cambian éstos a lo largo de la vida de una persona dando a entender que son aspectos que se comportan principalmente de manera estable. La teoría del establecimiento de metas enfatiza principalmente la importancia de la dimensión futuro cuando analiza la influencia del establecimiento de metas sobre la motivación de las personas como acicate para mantener y reforzar la dirección, persistencia e intensidad de los esfuerzos realizados para lograr alcanzar la meta fijada. La meta se constituye en un atractor regulador de las decisiones y acciones a llevar a cabo con el fin de alcanzarla, con lo cual la dinámica a estudiar queda atrapada en la trayectoria de acción que va desde la formulación de la meta hasta su consecución con independencia de los cambios que puedan acontecer en dicho trayecto y que podrían eventualmente cambiar la naturaleza original de la meta. En este caso la dinámica consiste de un relato episódico que describe el futuro más inmediato del comportamiento motivado, quedando pendiente qué ocurre cuando la meta es o no alcanzada (c.f. Fried \& Slowick, 2004; Locke \& Latham, 1990, 2002).

Las teorías de la justicia organizativa y más concretamente la clásica teoría de la equidad (Adams, 1963, 1965), en la que la motivación se entiende 
como un proceso de comparación social, tampoco encara la cuestión de sí las recompensas (y en este caso, también las aportaciones) varían o no a lo largo del tiempo y en el caso de que lo hicieran cómo serían estos cambios. Otro tanto ocurre con los nuevos desarrollos de estas elaboraciones teóricas, tal y como se puede observar en las propuestas de Cropanzano y Greenberg (1997) y de Leventhal (1980) sobre la justicia procedimental e interpersonal; todas ellas parecen no asumir el cambio en las comparaciones y sí, por paradójico que parezca, la naturaleza estática del proceso en la medida en que centran su interés en el desequilibrio (en este caso de percepciones entre aportes y recompensas) y en la necesidad de restablecer un balance que parece siempre conveniente y necesario. Lo que ocurre una vez reestablecido el equilibrio no ha interesado a estos autores.

En relación con la teoría social cognitiva, la noción de proceso es más dinámica al contemplar el papel de la realimentación en la conformación de la autoeficacia, piedra angular en esta teoría. En tanto creencia en las propias capacidades o limitaciones para enfrentar una situación determinada (un trabajo, una tarea, etc.), la autoeficacia emerge como proceso auto-referencial que se alimenta de las experiencias pasadas (propias o ajenas) y juega un papel central en la elección de tareas, en la predicción personal del desempeño y en la valoración del esfuerzo y la persistencia necesarias para llevarlas a cabo. Es decir, se trata de un proceso auto-realimentado que incorpora ya una dimensión de cambio y desarrollo a lo largo del tiempo. Sin embargo, también es necesario apuntar, tal y como lo señala Bandura (1995), que la autoeficacia al estar conformada de creencias es bastante estable por lo que los cambios en ella son más la excepción que la regla.

Tal y como lo plantean Navarro, Arrieta y Ballen (2007), gran parte de las teorías clásicas de procesos siguen compartiendo aspectos básicos de los modelos de reducción de tensión en tanto contemplan el comportamiento motivado como una forma de reducir alguna tensión (conseguir el resultado que se desea, conseguir la meta atractiva, re-equilibrar percepciones de inequidad, etc.), con lo cual se limita la posibilidad de que sea justamente lo contrario (el aumento de estimulación, la búsqueda de nuevos desequilibrios) lo que movilice el comportamiento.

\section{Modificaciones y cambios implementados en las teorías de proceso}

En los últimos tiempos y en buena parte debido a los cuestionamientos reseñados a lo largo de este trabajo, el campo de la motivación laboral ha estado sometido a revisiones críticas con el fin de adecuar las teorías y la investigación a las condiciones actuales. En este sentido, por ejemplo, el monográfico publicado por la Academy Management Review en 2004 destinado 
en forma exclusiva a motivación laboral y la reciente revisión publicada por Latham y Pinder en 2005 en el Annual Review of Psychology, son indicadores del creciente interés por renovar el campo a partir de la premisa de que es necesario dotar a la teoría de mayor profundidad y, en especial, de mayor dinamismo.

Esta revisión ha generado al menos dos líneas de trabajo: 1) la recomendación de expandir el abanico incorporando nuevas temáticas de investigación, y 2) someter a revisión las teorías mismas con el fin de analizar sus alcances y limitaciones. En cuanto a la primera línea, aparecen temáticas diversas como creatividad, grupos y cultura (Ambrose \& Kulik, 1999), trabajo en equipos, factores contextuales (características del puesto y ajuste persona-contexto), valores, factores preconscientes o subconscientes, aspectos interculturales (Latham \& Pinder, 2005), motivación y liderazgo en equipos virtuales, metas subconscientes, drogas psicotrópicas (Latham \& Ernst, 2006), motivación y procesos etarios (Kanfer \& Ackerman, 2004), componentes psicológicos y neurobiológicos de los afectos relacionados con el trabajo y que afectan la motivación (Seo, Barret \& Bartunek, 2004), identidad social en individuos y grupos y la motivación laboral (Ellemers, de Gilder \& Haslam, 2004), etc.

En cuanto a la segunda línea, son pocas las cosas a reseñar. Fried y Slowik (2004), por ejemplo, plantean la necesidad de integrar explícitamente el factor tiempo en la teoría del establecimiento de metas con la convicción de que como resultado de su incorporación se podría enriquecer significativamente la comprensión de tres de los componentes más importantes de dicha teoría, a saber, la dificultad de meta, el logro de meta y la especificidad de la meta, contribuyendo así al desarrollo de un modelo dinámico de la teoría del establecimiento de metas.

Locke y Latham (2004) brindan una serie de recomendaciones que, valoradas en conjunto, sugieren que es preciso reorientar la investigación dotándola de mayor profundidad y de mayor integración. En este sentido, proponen la necesidad de construir un megamodelo de la motivación que integre aquellas teorías más relevantes con el fin de potenciar la capacidad explicativa y predictiva de la teoría. A su vez recomiendan generar esfuerzos de apertura y flexibilización del campo merced a la integración y al intercambio con otras teorías y temáticas del campo de la psicología de las organizaciones (v.gr. efectividad en equipos, liderazgo, toma de decisiones, dirección estratégica) así como con otros aportes provenientes de otros campos de la psicología (v.gr. clínica, educativa, social).

Con respecto a la sugerencia de construir un megamodelo, Ambrose y Kulik (1999) varios años antes de que Locke y Latham (2004) propusieran esto, advirtieron que la elaboración de un modelo de tales proporciones podría resultar más contraproducente que beneficioso porque, por un lado, las teorías integradas podrían correr el peligro de perder su propia 
especificidad y, por otro lado, por su complejidad este modelo tendería a convertirse posiblemente en una especie de modelo del comportamiento organizacional por la cantidad de variables y relaciones que comportaría. Al observar el modelo integrador construido por Locke y Latham (ver figura 1) resulta difícil pasar por alto el señalamiento hecho por Ambrose y Kulik (1999).

Si bien es cierto hay que reconocer estos esfuerzos por promover la apertura y la renovación del campo, es posible identificar inconsistencias entre las recomendaciones brindadas por algunos de estos autores para hacer avanzar la teoría y la investigación y actuaciones posteriores. A modo de ejemplo, Latham y Locke (2004) plantean que es necesario establecer diálogos a nivel intra e interdisciplinario con el fin de fortalecer el campo y ampliar sus horizontes pero, paradójicamente, el mismo Latham en un artículo de revisión que escribe junto a Pinder (2005), y en el que pasan revista de la investigación realizada en los últimos 10 años en el campo de la motivación, no toman en cuenta trabajos llevadas a cabo desde otras concepciones alternativas a las tradicionales y que aparecen claramente consignadas en la base de datos PsycINFO de la APA. Por ejemplo, en su revisión no mencionan una serie de investigaciones abocadas al estudio dinámico de la motivación tales como las inspiradas en los trabajos de Csikszentmihalyi $(1990,1997)$ sobre flow (fluir) y motivación (Bakker, 2005; Delle Fave \& Massimini, 2004), los estudios de Guastello, Johnson y Rieke (1999) y de Guastello (2002) sobre la motivación como fenómeno caótico, la investigación de Navarro y Quijano (2003) sobre la construcción de un modelo de la motivación inspirado en las teorías de la complejidad.

Un análisis de esta omisión parece poner al descubierto al menos dos cosas: primera, que cuando se habla de "diálogo" es en referencia a ciertas teorías y enfoques que guardan relación con las teorías establecidas y, segunda, el sesgo de los autores que los lleva a omitir enfoques alternativos que ponen en cuestionamiento los alcances de las teorías tradicionales (ver apartado siguiente). En este sentido, parece que todavía ciertas críticas no logran permear lo suficiente como para llevar a los investigadores a cuestionarse algunos aspectos centrales de su quehacer. Es cierto que hay confrontaciones que mueven positivamente el campo provocando irritaciones allí donde las cosas se habían mantenido más o menos estables, sin embargo todavía resta profundizar más en los alcances de las críticas formuladas por ciertos autores. 
Figura 1.

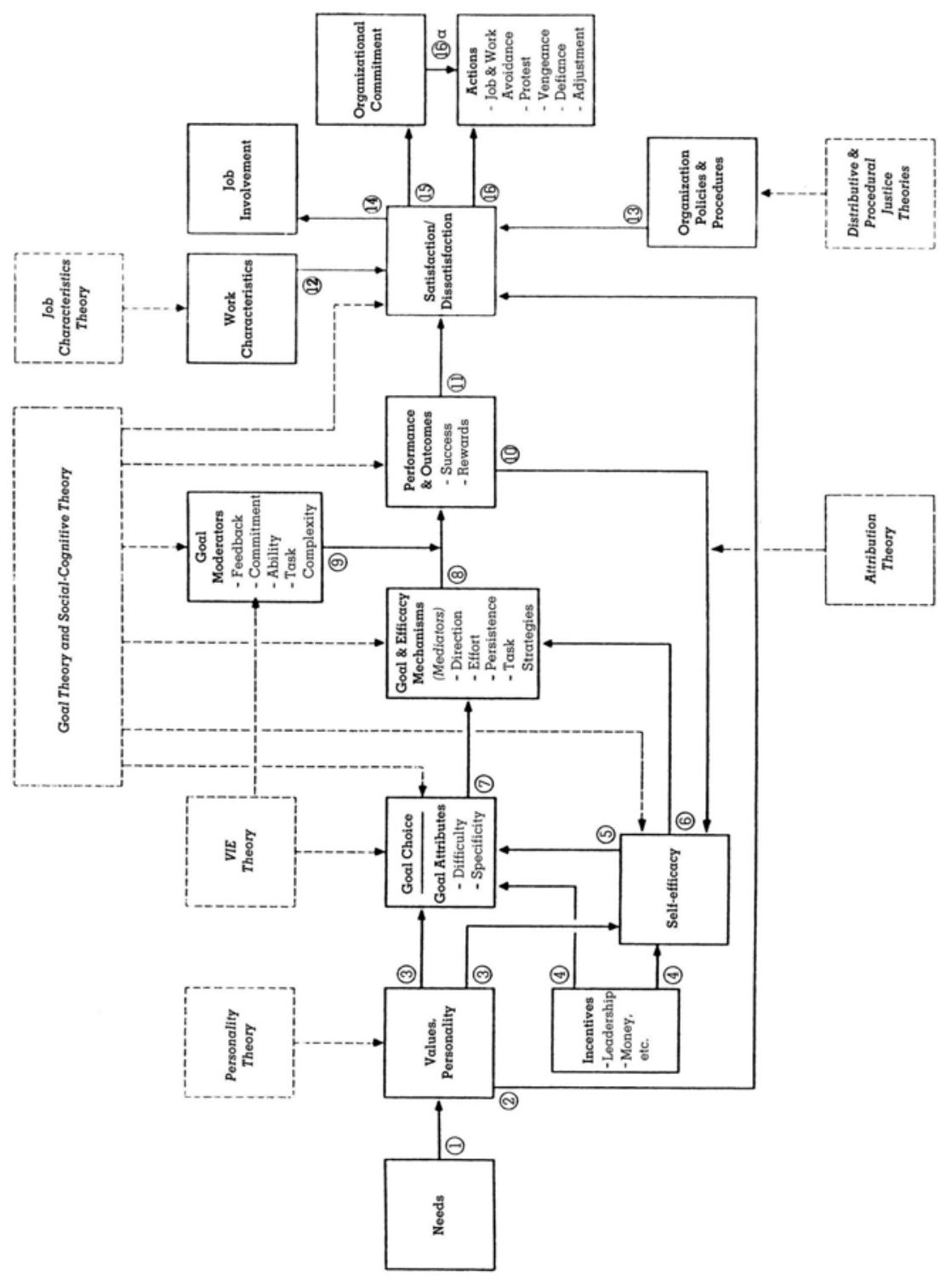

Figura. 1. Propuesta de modelo de motivación laboral de Latham y Locke conformado por la integración de diversas teorías de la motivación. Dentro de los cuadros con líneas punteadas aparecen las diferentes teorías tomadas en cuenta por los autores para elaborar la integración. Tomado de Locke y Latham (2004, p. 390) 


\section{Nuevos enfoques en el estudio de la motivación laboral}

$\mathrm{Al}$ analizar las propuestas planteadas con el fin de revitalizar el campo de la motivación extraña la falta de referencia a enfoques teóricos diferentes a los tradicionales, a pesar de que dichos enfoques han calado ya en otras áreas del comportamiento organizativo (por ejemplo, el enfoque constructivista es tenido en cuenta a la hora de abordar la estrategia, la cultura de empresa o el propio diseño organizativo; la complejidad se aplica para comprender el conflicto en las organizaciones y sus posibles efectos positivos sobre las mismas; etcétera). También llama la atención que los teóricos se abstengan de analizar críticamente los principios epistemológicos y metodológicos nucleares que sirven de plataforma a sus investigaciones. En general, las diferentes teorías formuladas para dar cuenta de la motivación en el trabajo se alimentan del paradigma positivista de la ciencia y del monismo metodológico que le caracteriza. En este sentido, en la mayor parte de las propuestas se puede observar que los principios fundamentales de que parten son la simplificación, el control, la causalidad y la predicción. Asimismo, prácticamente prima en ellos una perspectiva lineal a la hora de analizar el comportamiento de los fenómenos que estudian. Y probablemente ello explique en parte el estancamiento del campo, sobre todo en este momento en que las organizaciones, sus procesos y las personas que trabajan en ellas, están cambiando drásticamente su comportamiento a tenor con los cambios que están aconteciendo en el mundo. El control, la predicción y la estabilidad están cediendo terreno frente a la incertidumbre y dinamismo propios del espíritu de los tiempos. Y probablemente esto mismo explica la preocupación de incorporar en los modelos construidos el factor tiempo.

Tomando distancia de los enfoques tradicionales, la motivación en el trabajo ha empezado a ser estudiada desde nuevas perspectivas teórico metodológicas. La puesta en escena de otras teorías (v.gr. construccionismo, complejidad) y de otros enfoques metodológicos especialmente cualitativos (diarios de campo, observación de campo, simulación) empiezan a despuntar con relativa fuerza. En este sentido, los trabajos de Guastello, Johnson y Rieke (1999), de Navarro (2001), de Navarro y Quijano (2003) y de Navarro, Arrieta y Ballen (2007), son pioneros en el estudio del comportamiento dinámico de la motivación en el trabajo desde las teorías de la complejidad y el caos. Por su parte, los trabajos de Csikszentmihayli (1990) y colaboradores (Moneta \& Csikszentmihayli, 1996) inauguran también una línea de investigación de la motivación desde una perspectiva dinámica.

En el caso del trabajo de Moneta y Csikszentmihayli (1996), los autores se dan a la tarea de analizar las experiencias de fluir (flow) entendidas como 
experiencias de completa implicación en una actividad, es decir, como una intensa expresión subjetiva de absorción, disfrute y motivación intrínseca. A partir de los desarrollos de Csikszentmihayli, otros autores se han dedicado a investigar las experiencias de fluir en diferentes campos y con diferentes poblaciones como, por ejemplo, las experiencias de fluir en profesores (Bakker, 2005; Delle Fave \& Massimini, 2004), en recursos humanos (Salanova, Bakker, A. \& Llorens, 2006), y en actividades que podrían ser parte de tareas propias del trabajo como el uso de Internet (Chen, Wigand \& Nilan, 1999).

Con frecuencia para llevar a cabo estos estudios se apela al uso de metodologías y técnicas cualitativas como, por ejemplo, la denominada Experience Sampling Methodology (metodología de muestro de experiencias), constituida, tal y como lo señalan Conner et al. (2003) por un conjunto de métodos empíricos, especialmente la técnica de diario, que son diseñados para permitir a los participantes de una investigación documentar sus pensamientos, sentimientos y acciones fuera de los paredes del laboratorio y dentro del contexto de sus vidas cotidianas, es decir, dentro de lapsos de tiempo transcurridos mientras se desempeñan en escenarios naturales (c.f. Scollon et al., 2003; Feldman \& Barret, 2001).

En el caso de la investigación de Guastello y colaboradores (1999), los autores se dan a la tarea de estudiar el fluir (motivación intrínseca) obteniendo resultados que tienden a demostrar que muestra dinámicas no lineales y, más concretamente, una dinámica que se comporta en el tiempo de forma caótica. Para llevar a cabo este estudio los autores utilizaron la técnica de diario de campo con la que obtuvieron series temporales que les permitió detectar precisamente las turbulencias e inestabilidades del fenómeno.

Navarro (2001) y Navarro y Quijano (2003), por su parte, se dieron a la tarea de construir un modelo complejo para el estudio de la motivación integrando elementos extraídos tanto de las teorías de contenido como de las de procesos. En su modelo incluyeron variables ampliamente contrastadas en la investigación tradicional (motivos, percepción de autoeficacia y percepción de instrumentalidad) obteniendo unos resultados que muestran que el modelo propuesto es estable sólo cuando el patrón de cambio en la variable motivos es caótico y que en los demás casos se producen dinámicas explosivas (los valores de todas la variables crecen hasta el infinito) o implosivas (los valores de todas las variables decrecen hasta cero) que resultan inverosímiles. De acuerdo con estos resultados, al igual que en el caso de la investigación de Guastello y colaboradores, los autores encuentran comportamientos complejos en la motivación y proponen que es necesario estudiar este fenómeno y entender su dinámica prestando especial atención a los flujos continuos de realimentación y sugieren que un modelo complejo de la motivación, a diferencia del megamodelo propuesto por Locke y 
Latham (2004), no requiere de un gran número de variables sino de la selección de aquellas realmente significativas para los sujetos y del análisis de las relaciones dinámicas que se establecen entre ellas.

Figura 2.

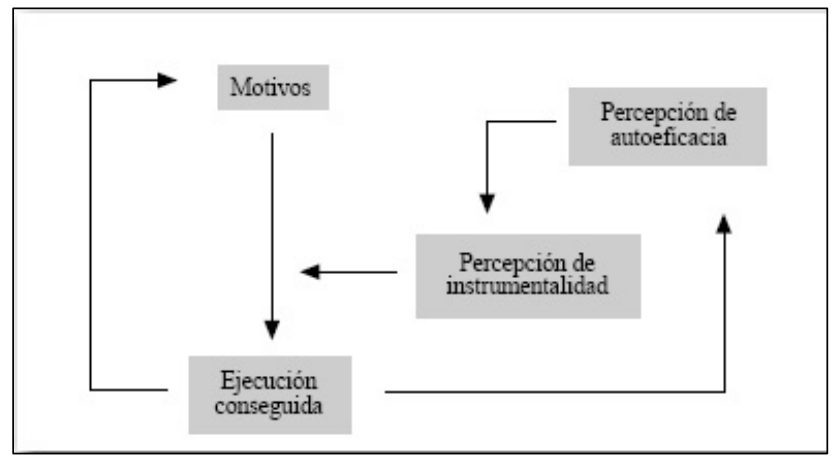

Fig. 2. Modelo complejo de motivación en el trabajo. Tomado de Navarro y Quijano (2003, p. 645)

Posteriormente Navarro, Arrieta y Ballen (2007), llevaron a cabo un estudio con el fin de analizar a nivel descriptivo la dinámica de la motivación laboral. Según los resultados de esta investigación las dinámicas encontradas se comportan en su mayoría de forma no lineal con un porcentaje muy elevado de dinámicas moviéndose entre la no linealidad y el azar. En concordancia con lo anterior, la mayor parte de las series temporales relativas a las variables analizadas no muestran estacionalidad alguna mientras que el comportamiento de la motivación parece indicar que ésta no se detiene nunca y tampoco parece estar a la espera de estimulación externa para que se active el comportamiento motivado.

Vistos en conjunto, los resultados comunicados en los trabajos reseñados anteriormente permiten sacar provisionalmente una serie de conclusiones. En primer lugar, que la motivación laboral no es un proceso tan estable como se ha pensado hasta el momento y como ha sido considerado desde el punto de vista de las teorías tradicionales. Segundo, que es muy probable que los resultados obtenidos en los enfoques tradicionales se deben en parte a los diseños metodológicos empleados (estudios transversales, cuestionarios y escalas aplicados esporádicamente, etc.) y que si se utilizasen otro tipo de diseños (longitudinales o de simulación) los resultados con gran probabilidad serían diferentes. Y tercero, y como conclusión obvia, que es posible estudiar fructíferamente la motivación laboral empleando enfoques y modelos alternativos. 


\section{Conclusiones}

En los albores del nuevo milenio parece haber consenso en cuanto a que el estudio de la motivación laboral se encuentra en una fase crítica debido principalmente a la falta de renovación y a las limitaciones de las teorías tradicionales para responder a las transformaciones que están experimentando las organizaciones y el mundo del trabajo. La reconocida necesidad de abrir el campo de la investigación a nuevos modelos y enfoques pasa a nuestro juicio principalmente por analizar críticamente los presupuestos epistemológicos y metodológicos sobre los que se ha sustentado la investigación tradicional, de lo contrario los cambios que se están dando y los que habrán de llegar en el futuro no pasarán de ser intentos revisionistas con el objetivo de alargar indefinidamente la vigencia de un conjunto de teorías que se niegan a perder el dominio que han tenido durante mucho tiempo. Ello no significa en modo alguno que el conocimiento acumulado hasta el momento resulte inútil y que se deba prescindir de las teorías existentes, lo que quiere significar es que dadas las limitaciones identificadas se hace preciso encarar la tarea de adecuar los enfoques existentes a las nuevas condiciones y de desarrollar nuevas propuestas a nivel teórico y metodológico que contribuyan, tal y como lo mencionan Steers, Mowday y Shapiro (2004), a empujar el campo hacia un nuevo nivel de entendimiento.

Las investigaciones en motivación laboral llevadas a cabo desde el paradigma de la complejidad, a pesar de su número limitado, han demostrado consistentemente que cuando la motivación laboral es estudiada realmente desde una perspectiva dinámica, es decir, utilizando diseños que permitan observar sistemáticamente y a lo largo del tiempo el comportamiento del fenómeno tomando en cuenta los procesos de realimentación y analizando los datos recabados con técnicas adecuadas, los resultados revelan la presencia de dinámicas no lineales en el comportamiento del fenómeno que distan de guardar relación con la estabilidad y regularidad que le han sido atribuidas tradicionalmente a este fenómeno y de las que generalmente se parte a la hora de investigarlo. Es probable que la implementación de diseños de investigación más dinámicos en otros paradigmas permita arribar a resultados similares.

Algunos podrían argumentar que las nuevas estrategias de análisis estadístico, especialmente los análisis multinivel y los modelos de ecuaciones estructurales, permiten realizar análisis de mayor complejidad y dinamismo en comparación con diseños correlacionales más sencillos, lo cual sin duda es cierto. Sin embargo el problema sigue radicando en el tipo de datos con los que se trabaja, esto es, se pueden utilizar técnicas más dinámicas pero no por ello deja de ser un contrasentido pretender "atrapar" el dinamismo de los fenómenos a partir de unas pocas observaciones de su comportamiento 
(cuando no solo de una). Y, por otra parte, cuando se analizan los modelos teóricos construidos rápidamente se puede observar que la relación unidireccional establecida entre las diversas variables no tiene en cuenta los procesos de realimentación característicos de los fenómenos humanos, esto es, no son capaces - por su propia lógica- de evaluar los procesos de afectación recíproca.

Para finalizar, es de destacar que en los últimos tiempos en este campo se estén suscitando confrontaciones paradigmáticas alimentadas en parte por la toma de conciencia acerca de las limitaciones inherentes a los modelos teóricos desarrollados hasta el momento para estudiar y explicar la motivación en el trabajo, y en parte también por las nuevas condiciones y exigencias de un contexto que cambia aceleradamente y que plantea nuevos retos a la investigación y a la gestión. Queda seguir profundizando en las críticas formuladas y dirigir la discusión no solo desde las teorías existentes hasta el momento sino principalmente desde las propias condiciones del objeto de estudio.

\section{Referencias}

Adams, J. S. (1963). Toward an understanding of inequity. Journal of Abnormal Psychology, 67, $422-436$.

Adams, J. S. (1965). Inequity in social exchange. En L. Berkowitz (Ed.), Advances in experimental social psychology (pp.267-296). New York: Academic Press.

Ambrose, M. L. \& Kulik, C. T. (1999). Old friends, new faces: Motivation research in the 1990s. Journal of Management, 25, 231-292.

Ancona, D. G., Goodman, P. \& Tushman, M. (2001). Time: a new research lens. Academy of Management Review, 26 (4), 645-663.

Ancona, D. G., Okhuysen, G. \& Perlow, L. (2001). Taking time to integrate temporal research. Academy of Management Review, 26 (4), 512-529.

Andriessen, J. H. (1994). Conditions for successful, adoption and implementation of telematics in user organizations. En J.H. Andriessen y R.A. Roe (eds.), Telematics and Work (pp. 411-439). Hillsdale, USA: Lawrence Erlbaum Associates.

Andriessen, J. H. (2002). Working with groupware. Understanding and evaluating collaboration technology. London: Springer.

Bakker, A. (2005). Flow among music teachers and their students: The crossover of peak experiences. Journal of Vocational Behavior, 66, 26-44.

Bandura, A. (Ed.) (1995). Self-efficacy in changing societies. New York: Cambridge University Press.

Bluedorn, A. C. \& Denhardt, R. B. (1988). Time and organizations. Journal of Management, 14 (2), 299-320.

Campbell, J. P., Dunnette, M. D., Lawler, E. E. \& Weick, K. (1970). Managerial performance and effectiveness. New York: McGraw-Hill. 
Campbell, J. P. \& Pritchard R. D. (1976). Motivational theory in industrial and organizational psychology. En M.D. Dunnette (Ed.), Handbook of Industrial and Organizacional psychology (pp. 63-130). Chicago: Rang McNally

Chen, H., Wigand, R. T. y Nilan, M. S. (1999). Optimal experience of Web activities. Computers in Human Behavior, 15, 585-608.

Conner, T. L., Feldman Barrett, L., Bliss-Moreau, E., Lebo, K. \& Kaschub, C. (2003). A practical guide to experience-sampling procedures. Journal of Happiness Studies, 4, 53-78.

Cropanzano, R. \& Greenberg, J. (1997). Progress in organizational justice: tunneling through the maze. En C. L. Cooper y I. T. Robertson (Eds.), International Review of Industrial and Organizational Psychology (Vol 12, pp. 317-372). New York: Wiley.

Csikszentmihalyi, M. (1990). Flow: the psychology of optimal experience. New York: Harper Collins.

Csikszentmihalyi, M. (1997). Finding flow: The psychology of engagement with everyday life. New York: HarperCollins.

Delle Fave, A. \& Massimini, F. (2004). The cross-Cultural Investigation of Optimal Experience. Ricerche di Psicologia, 27, 79-103.

Ellemers, N., De Gilder, D. \& Haslam, S. A. (2004). Motivating individuals and groups at work: a social identity perspective on leadership and group performance. Academy of Management Review, 29 (3), 459-478.

Feldman Barrett, L. \& Barrett, D. J. (2001). An introduction to computerized experience sampling in psychology. Social Science Computer Review, 19 (2), 175-185.

Fried, Y. \& Slowik, L. H. (2004). Enriching goal-setting theory with time: an integrated approach. Academy of management Review, 29 (3), 494-422.

George, J. M. \& Jones, G. R. (2000). The role of time in theory and theory building. Journal of Management, 26 (4), 657-684

Guastello, S. J. (2002). Managing emergent phenomena. Nonlinear dynamics in work organizations. London: Lawrence Erlbaum Associates Publishers.

Guastello, S. J., Johnson, E. A. \& Rieke, M. L. (1999). Nonlinear dynamics of motivational flow. Nonlinear Dynamics, Psychology, and Life Sciences, 3 (3), 259-273.

Kanfer, R. (1990). Motivational theory and industrial and organizational psychology. En M. Dunnette y L. M. Hough (Eds.), Handbook of Industrial and Organizational psychology ( ${ }^{\mathrm{a}} \mathrm{ed}$., Vol. 3, pp. 269-313). Palo Alto, CA: Consulting Psychologists Press.

Kanfer, R. \& Ackerman, P. (2004). Aging, adult development, and work motivation. Academy of Management Review, 29 (3), 440-458.

Latham, G.P \& Ernst, C. T. (2006). Keys to motivating tomorrow's workforce. Human Resource Management Review, 16, 181-198.

Latham, G. P. \& Pinder, C. C. (2005). Work motivation theory research at the down of the twenty-first century. Annual Review of Psychology, 56, 485-516.

Leventhal, G. S. (1980). What should be done with equity theory? En K. J. Gergen, M. S. Greenberg y R. H. Willis (Eds.), Social exchange: advances in theory and research (pp. 27-55). New York: Plenum.

Locke, E. A. \& Latham, G. P. (1990). A theory of goal setting and task performance. Englewood Cliffs, NJ: Prentice-Hall.

Locke, E. A. \& Latham, G. P. (2002). Building a practically usefull theory of goal setting and task motivation: A 35-year odyssey. American Psychologist, 57, 705-717.

Locke, E. A. \& Latham, G. P. (2004). What should we do about motivation theory? Six recommendations for the twenty-first century. Academy of Management Review, 29 (3), 388403.

McGrath, J. E. \& Kelly, J. R. (1986). Time and Human Interaction. New York: Guilford Press.

McGrath, J. E. \& Tschan, F. (2004). Temporal matters in social psychology. Examining the role of time in the lives of groups and individuals. Washington DC: APA Publications. 


\section{Carlos Arrieta-Salas y José Navarro-Cid}

Mitchell, T. \& James, L. (2001). Building better theory: time and the specification of when things happen. Academy of Management Review, 26 (4), 530,547.

Moneta, G. B. \& Csikszentmihalyi, M (1996). The effect of perceived challenges and skills on the quality of subjective experience. Journal of Personality, 64, 275-310.

Morgan, G. (1999). Imagin-i-zación. Una nueva aptitud crucial para la conducción y el management en un mundo en movimiento y cambio. Barcelona: Ediciones Granica S.A.

Mosakowski, E. \& Earley, P.C. (2000). A selective review of time assuptions in strategy research. Academy of Management Review, 25 (4), 796-812.

Mowday, R. T. \& Sutton, R. I. (1993). Organizational Behavior: Linking individual and groups to organizational contexts. Annual Review of Psychology, 44, 195-229.

Munduate, L. (1994). Motivación y compromiso en el trabajo. En J. Ma . Peiró y J. Ramos (Dirs.), Intervención psicosocial en las organizaciones (pp. 203-258).Barcelona: PPU.

Munné, F. (1995). Las teorías de la complejidad y sus implicaciones en las ciencias del comportamiento. Revista Interamericana de Psicología, 29 (1), 1-12.

Navarro, J. (2001). Las Organizaciones como Sistemas Abiertos Alejados del Equilibrio. Tesis Doctoral. Universitat de Barcelona (España).

Navarro, J. \& Quijano, S. (2003). Dinámica no lineal en la motivación en el trabajo: propuesta de un modelo y resultados preliminares. Psicothema, 15 (4), 643-649.

Navarro, J., Arrieta, C. \& Ballen, C. (2007). An approach to the study of the dynamics of work motivation using diary method. Nonlinear Dynamics, Psychology, and Life Sciences, 11(4), 473-498.

Peiró, J. M. (1990). Organizaciones: nuevas perspectivas psicológicas. Barcelona: PPU.

Prieto, F., Zornoza, A., Orengo, V. \& Peiró, J.M. (1996). Trabajo y nuevas tecnologías de la información. En: Peiró, J.M. y Prieto, F. (Eds.), Tratado de Psicología del Trabajo. La actividad laboral en su contexto. (pp. 97-127). Madrid: Síntesis.

Prieto, F., Zornoza, A. \& Peiró, J.M. (1996). Nuevas tecnologías de la información en la empresa: Una aproximación psicosocial. Madrid: Ediciones Pirámide S.A.

Robbins, S. J. (2003). Organizational behavior (10 th ed.). Upper Saddle River, NJ: Prentice Hall.

Roe, R.. A. (2005a). No more variables, please. Giving time a place in work and organizational psychology. En H. Kepir Sinangil, F. Avallone y A. Caetano (Eds.), Convivence in Organizations and Society (pp. 11-20). Milán: Guerini.

Roe, R.. A. (2005b). Studying time in organizational behavior. Maastricht Research School of Economics of Technology and Organization. Research Memoranda, número 048. Recuperado el 10-12-2007 de : http://edocs.ub.unimaas.nl/loader/file.asp?id=1120.

Rousseau, D. M. (1997). Organizational Behavior. Annual Review of Psychology, 48, 515-546.

Salanova, M., Hontangas, P. \& Peiró, J. M. (1996). Motivación Laboral. En J.M.Peiró y F. Prieto (Eds), Tratado de psicología del trabajo (pp. 215-249). Madrid: Editorial Síntesis.

Salanova, M., Bakker, A. \& Llorens, S. (2006). Flow at Work: Evidence for a Gain Spiral of Personal and Organizational Resources. Journal of Happiness Studies, 7, (1), 1-22.

Scollon, C. H., Kim-Prieto, C. \& Diener, E. (2003). Experience sampling: promises and pitfalls, strengths and weaknesses. Journal of Happiness Studies, 4 (5), 5-34.

Seo, M. G., Barrett, L. F. \& Bartunek, J. M. (2004) The role of affective experience in work motivation. Academy of Management Review, 29 (3), 423-439.

Steers, R. M., Mowday, R. T. \& Shapiro, D.L. (2004). The future of work motivation theory. Academy of Management review, 29 (3), 379-387.

Thierry, H. (1998). Motivation and satisfaction. En P. Drenth, H. Tierry y Ch. de Wolf (Eds.), Handbook of work and organizational psychology. Organizational psychology (Vol. 4, 2a ed., pp. 253-289). Hove, England: Psychology Press.

Vroom, V. H. (1964). Work and motivation. New York: Wiley

Weick, K. E. (2001). Making sense of the organization. Malden, MA: Blackwell Publishing.

Wilpert, B. (1995). Organizational Behavior. Annual Review of Psychology, 4, 59-90. 


\section{Motivación en el trabajo: viejas teorias, nuevos horizontes}

Wright, T. A. (2002). Dialogue: The importance of time in organizational research. Academy of Management Review, 45, 343-345.

Zaheer, S., Albert, S. \& Zaheer, A. (1999). Time scales and organizational theory. Academy of Management Review, 24 (4), 725-741.

Zornoza, A., Orengo, V. \& Peiró, J.M. (2000). Implicaciones de las tecnologías de la información para el trabajo y las organizaciones. Una perspectiva psicosocial. Revista Gallega de Empleo, 1, 91-141.

Recibido: 21 de abril de 2007 Aceptado: 21 de diciembre de 2007 